\title{
Dynamical and chemical evolution of NGC 1569
}

\author{
S. Recchi ${ }^{1}$, G. Hensler ${ }^{1}$, L. Angeretti ${ }^{2}$, and F. Matteucci ${ }^{3}$ \\ ${ }^{1}$ Institute of Astronomy, Vienna University, Türkenschanzstrasse 17, 1180 Vienna, Austria \\ e-mail: recchi@astro.univie.ac.at \\ 2 INAF - Osservatorio Astronomico di Bologna, via Ranzani 1, 40127 Bologna, Italy \\ 3 Dipartimento di Astronomia, Università di Trieste, via GB Tiepolo, 11, 34131 Trieste, Italy
}

Received 16 May 2005 / Accepted 7 September 2005

ABSTRACT

Blue Compact Dwarf and Dwarf Irregular galaxies are generally believed to be unevolved objects, due to their blue colors, compact appearance and large gas fractions. Many of these objects show an ongoing intense burst of star formation or have experienced it in the recent past. By means of 2-D hydrodynamical simulations, coupled with detailed chemical yields originating from SNeII, SNeIa, and intermediate-mass stars, we study the dynamical and chemical evolution of model galaxies with structural parameters similar to NGC 1569, a prototypical starburst galaxy. A burst of star formation with short duration is not able to account for the chemical and morphological properties of this galaxy. The best way to reproduce the chemical composition of this object is by assuming long-lasting episodes of star formation and a more recent burst, separated from the previous episodes by a short quiescent period. The last burst of star formation, in most of the explored cases, does not affect the chemical composition of the galaxy, since the enriched gas produced by young stars is in a too hot phase to be detectable with the optical spectroscopy. Models assuming the infall of a big cloud towards the center of the galaxy reproduce the chemical composition of the NGC 1569, but the pressure exercised by the cloud hampers the expansion of the galactic wind, at variance with what observed in NGC 1569.

Key words. hydrodynamics - ISM: abundances - ISM: jets and outflows - galaxies: evolution - galaxies: individual: NGC 1569

\section{Introduction}

Among dwarf galaxies, Blue Compact Dwarfs (BCDs) and Dwarf Irregulars (dIrrs) are characterized by large gas content and often active star formation (SF). They also show very blue colors and low metallicities and are therefore commonly believed to be systems similar to primeval galaxies. They are consequently ideal targets to study the feedback between star formation and interstellar medium. They have also been suggested to be the local counterparts of faint blue objects (Babul \& Rees 1992; Lilly et al. 1995; Ellis 1997).

It has recently become clear that most of these objects show the presence of at least intermediate age stars (Kunth \& Östlin 2000), but their contribution to the global metallicity and energy budget of the galaxy is still unknown. It is interesting to simulate galaxies whose light and colors are dominated by young stars and to see whether their chemical and morphological properties can be explained by a recent burst of star formation or whether older episodes of SF are required.

In general, the SF in BCDs is described as a bursting process (Searle et al. 1973), namely, short, intense episodes of $\mathrm{SF}$ are separated by long inactive periods. A gasping mode of SF (long episodes of SF of moderate intensity separated by short quiescent periods) is instead often used to describe the star formation in dIrrs (Aparicio \& Gallart 1995). Galaxy candidates experiencing gasping SF are for instance NGC 6822
(Marconi et al. 1995), Sextans B (Tosi et al. 1991), and the LMC (Gallagher et al. 1996). These two different SF modes have been studied in the framework of chemical evolution models (Bradamante et al. 1998; Chiappini et al. 2003a; Romano et al. 2005a), and they produce similar results, because the chemical enrichment mostly depends on the gas consumption. It is therefore not easy to discriminate between these two different SF scenarios on the basis of chemical evolution models alone. Moreover, the chemical evolution of galaxies changes under conditions imposed by dynamical processes. Therefore, a chemo-dynamical approach is needed.

In the context of a long term project aimed at studying the dynamical and chemical evolution of dwarf galaxies, in this paper we present the results on NGC 1569, a gas-rich, metalpoor and possibly young dwarf galaxy. In the following, we briefly summarize the main results presented in a series of papers (Recchi et al. 2001, 2002, 2004) where we have analyzed the dynamical and chemical evolution of IZw18, the most metal-poor galaxy locally known. Although these models were tailored on this specific object, they give a hint on the enrichment of a galaxy in the first hundreds of Myr of evolution, and in addition, IZw18 will become useful in our discussion. Thus, the main results are:

- A galactic wind develops in almost all the models. This wind carries away mostly the metals freshly produced during the burst. 
- SNeIa are important triggerers of the galactic wind and the metals produced by this kind of SNe (mainly iron-peak elements) are ejected more easily than the products of SNeII (mostly $\alpha$-elements).

- In models with a bursting SF, the freshly produced metals cool down in a short time-scale (of the order of a few $10^{7} \mathrm{yr}$ ). This can justify the so-called "instantaneous mixing" approximation, commonly assumed in chemical evolution models (Matteucci 1996) and apparently confirmed by recent observations of the most metal-poor stars in the Galactic halo (Spite et al. 2005; Arnone et al. 2005). In models with a gasping SF instead, the metals produced by the last burst of SF mix with the surrounding ISM in a much longer time-scale.

- Either by assuming a gasping SF scenario or a bursting one, no stars older than 300-500 Myr are required in order to reproduce the characteristics of IZw18.

In summary, a significant contribution of intermediate-mass stars is decisive in the chemical and dynamical evolution of IZw18, but no old stars are required. From a chemodynamical point of view, IZw18 is therefore a young galaxy. Recently, Izotov \& Thuan (2004) showed that, although their data go 1-2 mag deeper than the tip of the Red Giant Branch phase, no stars are detected in this stage of their evolution. This sets a robust constraint on the age of this galaxy (of the order of a few hundred Myrs) and lets them conclude that IZw18 is a bona fide young galaxy (at least young in a cosmological sense), in agreement with the chemodynamical calculations and at variance with recent claims that most of the Blue Compact Dwarf galaxies (including IZw18) host a very old (with an age of several Gyrs) population of stars (e.g. Östlin 2000). It is however worth pointing out that new analyses of the Izotov \& Thuan (2004) ACS data (Momany et al. 2005; Tosi, private communication) show probably the presence of RGB stars in IZw18, therefore this object might be older than assumed by Izotov \& Thuan.

The subject of this paper, NGC 1569, is often classified as a DIrr, whereas IZw18 is a BCD galaxy. Despite the different classification, these two objects show similar properties: both are in the aftermath of an intense burst of SF, are very metal poor and have an extremely large gas content. NGC 1569 is located near the Local Group. Its distance is $2.2 \mathrm{Mpc}$ (Israel 1988 ) but different values can be found in literature. It is also characterized by a significant extinction $(E(B-V)=0.56$; Israel 1988), due to its low galactic latitude. Its stellar population is dominated by three super star clusters (de Marchi et al. 1997; Origlia et al. 2001), but a large number of star clusters of smaller dimensions has been detected (Anders et al. 2004). The total dynamical mass of NGC 1569 is $M_{\text {dyn }} \sim 3.3 \times 10^{8} M_{\odot}$ (Israel 1988), one third of which is neutral hydrogen (Stil \& Israel 2002).

The oxygen abundance of NGC 1569 is $12+\log (\mathrm{O} / \mathrm{H})=$ $8.19 \pm 0.02$ (Kobulnicky \& Skillman 1997). If oxygen is assumed to be a tracer of the global metallicity, it implies a global metallicity of $\sim 0.3 Z_{\odot}$ (assuming the Asplund et al. 2005 solar chemical composition; a value of $\sim 0.2 Z_{\odot}$ is obtained adopting the Anders \& Grevesse 1989 one). The N/O ratio lies $\sim 0.2$ dex above the average $\log (\mathrm{N} / \mathrm{O})$ detected in the most metal-poor galaxies $(\overline{\log (\mathrm{N} / \mathrm{O})} \sim-1.6$; Izotov \& Thuan 1999). According to Kobulnicky \& Skillman (1997), $\log (\mathrm{N} / \mathrm{O})=-1.39 \pm 0.05$. These observations revealed also a global uniformity of the chemical composition of NGC 1569, indicating either an exceptionally fast mixing of the freshly produced metals or that the youngest generations of stars are not contributing to the global metallicity budget of this galaxy.

Outflowing gas from NGC 1569 has been inferred from the kinematics of an extended system of $\mathrm{H} \alpha$ filaments (Heckman et al. 1995; Martin 1998) and from a diffuse X-ray emission (Heckman et al. 1995; Martin, Kobulnicky \& Heckman 2002; Ott, Walter \& Brinks 2005). Martin et al. (2002) attempted to evaluate the chemical composition of the hot medium, obtaining metallicities larger than those in the H II regions (but see also Ott et al. 2005, in which a good fit is obtained when assuming metallicities of the hot gas similar to the ones measured in the H II regions). Finally, there is observational evidence of the presence of extended H I clouds and complexes surrounding NGC 1569 (Stil \& Israel 1998). In particular, there is a series of gas clumps in the southern halo probably connected with a H I arm present in the western side of the galaxy. These H I features can be attributed to the debris of a tidally disrupted big cloud infalling towards NGC 1569 (Mühle et al. 2005).

The aim of this paper is to study the dynamical and chemical evolution of NGC 1569, by performing 2-D hydrodynamical simulations in cylindrical coordinates, coupled with detailed chemical yields coming from SNeII, SNeIa, and winds from intermediate-mass stars (IMS). In this context, we study the case of a single, dominating burst of star formation and of a more complex history of star formation to investigate which scenario better reproduces the chemical composition of this object. We will also analyze the chemical composition of different gas phases and we will finally study the impact of the infall of cold H I clouds on the chemical and energetic budget of the galaxy. In Sect. 2 we summarize our knowledge about the stellar component and the past star formation history of NGC 1569. In Sect. 3 we briefly summarize the model and the adopted assumptions in the simulations. Results are presented in Sect. 4 and, finally, some conclusions are drawn in Sect. 5.

\section{The star formation history of NGC 1569}

The stellar component of NGC 1569 is dominated by two super star clusters, usually called "A" and "B" (nomenclature of Arp \& Sandage 1985) although SSC-A has found to be a double system (De Marchi et al. 1997). The two complexes SSC-A and SSC-B are located $\sim 8^{\prime \prime}(\sim 80$ pc assuming a distance of $2.2 \mathrm{Mpc}$ ) apart. Their mass is estimated to be (516) $\times 10^{5} M_{\odot}$ and their age of the order of a few tens Myrs (Anders et al. 2004). Although young stars dominate the light of NGC 1569, Hubble Space Telescope (HST) observations revealed the presence of older stars. Vallenari \& Bomans (1996) found that NGC 1569 experienced a recent episode of SF, lasting about $100 \mathrm{Myr}$ and terminating $4 \mathrm{Myr}$ ago. They also found hints for an older and weaker episode of SF, from 1.5 Gyr until 150 Myr ago. Greggio et al. (1998) found a SF constant over the last 100 Myr with little or no quiescent periods. The SF 
rate they infer was of the order of $0.5-3 M_{\odot} \mathrm{yr}^{-1}$, depending on the adopted IMF slope. Martin et al. (2002), by fitting the $\mathrm{H} \alpha$ luminosity, found a much weaker SF rate of $0.16 M_{\odot} \mathrm{yr}^{-1}$ for the last episode of SF. Aloisi et al. (2001) found an age gradient in NGC 1569, in the sense that the youngest stars are mostly concentrated in the 3 super star clusters, intermediateage stars are almost uniformly distributed, whereas oldest stars are mostly located at the outskirts of the starbursting regions.

Recently Angeretti et al. (2005, hereafter A05), by analyzing HST's NICMOS/NIC2 data in a field of $200 \times 200 \mathrm{pc}^{2}$ (encompassing the 3 largest star clusters), concluded that, in order to reproduce the Color-Magnitude Diagrams (CMDs) of NGC 1569 with synthetic ones, assuming a Salpeter IMF between 0.1 and $120 M_{\odot}$, the best choice of the SF history is:

- A most recent episode of SF, from 37 to $13 \mathrm{Myr}$ ago, at a rate of $0.13 M_{\odot} \mathrm{yr}^{-1}$ (equivalent to $3.2 M_{\odot} \mathrm{yr}^{-1} \mathrm{kpc}^{-2}$ ).

- An intermediate episode of SF, commencing $150 \mathrm{Myr}$ ago and finishing $40 \mathrm{Myr}$ ago (therefore separated from the recent episode of SF by a very short quiescent period of $3 \mathrm{Myr}$ ), at a rate of $0.04 M_{\odot} \mathrm{yr}^{-1}$ (equivalent to $1 M_{\odot} \mathrm{yr}^{-1} \mathrm{kpc}^{-2}$ ).

- An older episode of SF, ending 300 Myr ago (therefore implying 150 Myr of inactivity between this episode and the intermediate one) and commencing either $600 \mathrm{Myr}$ ago (at a rate of $0.05 M_{\odot} \mathrm{yr}^{-1}$, equivalently $1.2 M_{\odot} \mathrm{yr}^{-1} \mathrm{kpc}^{-2}$ ), or $2 \mathrm{Gyr}$ ago (at a rate of $0.01 M_{\odot} \mathrm{yr}^{-1}$, equivalently $0.25 M_{\odot} \mathrm{yr}^{-1} \mathrm{kpc}^{-2}$ )

It is worth pointing out that A05 cannot exclude a low SFR during the inter-burst phases and in the last 13 Gyr. In addition, the history of SF derived in the NICMOS area studied by A05 could differ from that of the external parts mainly because of the stochastic origin of the SF.

\section{The model}

\subsection{The gravitational potential and the initial gas configuration}

We simulate a galaxy model resembling NGC 1569 by means of a 2-D hydro code in cylindrical coordinates. It is worth pointing out that NGC 1569 is characterized by a pronounced arm of gas in the western side and by an extensive system of $\mathrm{H} \alpha$ filaments, therefore it is definitely non-axisymmetric. A 2-D geometry can treat the global properties of the galaxy but not the complex H I features in detail (see also Sect. 4.7). The ISM is initially isothermal and in hydrostatic equilibrium with the galactic potential well and the centrifugal force. The galactic potential well is given by a quasi-isothermal spherical dark halo and an oblate stellar component. The dark halo has a core radius of $1 \mathrm{kpc}$ and is truncated at a radius of $20 \mathrm{kpc}$. Its total mass is $1.4 \times 10^{9} M_{\odot}$ but only a very small fraction of this dark matter $(\sim 5 \%)$ is within the optical size of NGC 1569 . The estimates of the total mass by Reakes (1980) point towards a lower value $\left(\sim 5 \times 10^{8} M_{\odot}\right)$ but, as pointed out by Martin (1998), the relative contribution of dark and baryonic matter, at least in the central region of the galaxy, is poorly constrained.
As already described in Recchi et al. (2001), in order to reproduce a gas distribution with the correct ratio between minor and major axis ( 0.5; Reakes 1980), we introduce a stellar component described by an oblate King profile. Due to the relatively low mass, compared with the total mass of the galaxy, the contribution of the newly born stars to the total potential well is neglected and therefore the potential is fixed in time. The details on how to build a rotating gas distribution in equilibrium with a given potential well can be found in D'Ercole \& Brighenti (1999).

\subsection{Chemistry and supernovae}

We couple our hydrodynamical simulations with detailed chemical yields originating from SNeII, SNeIa, and winds from IMS. The SNeIa rate is calculated according to the so-called Single-Degenerate scenario (namely $\mathrm{C}-\mathrm{O}$ white dwarfs in binary systems that explode after reaching the Chandrasekhar mass because of mass transfer from a red giant companion). It is worth noticing that, in the case of episodes of SF of short duration, a significant number of SNeIa explodes after a timescale of the order of $10^{8} \mathrm{yr}$ (Matteucci \& Recchi 2001), therefore they can alter the chemical composition of the galaxy in a timescale much shorter than often invoked for this kind of SNe (of the order of $1 \mathrm{Gyr}$ ). In the so-called Double-Degenerate scenario (explosion after merging of two white dwarfs as a result of angular momentum losses via gravitational wave radiation) one has to take into account the delay due to the emission of gravitational waves. However, in the case of instantaneous bursts of star formation, also the Double-Degenerate scenario predicts a peak in the SNeIa rate after 100-200 Myr (see e.g. Ruiz-Lapuente \& Canal 1998).

In order to mimic continuous episodes of SF, we calculate the mass of stars formed between $t$ and $t+\Delta t$, with $\Delta t=10^{5} \mathrm{yr}$. This Stellar Population (SP) is treated as a starburst. We calculate the evolution, in space and time, of 8 chemical elements of particular astrophysical relevance, namely $\mathrm{H}, \mathrm{He}, \mathrm{C}, \mathrm{N}, \mathrm{O}, \mathrm{Mg}$, $\mathrm{Si}, \mathrm{Fe}$. The mass return rate from each of these chemical elements is calculated according to Eq. (1) of Recchi et al. (2004), namely we sum up the contributions of all the SPs which can provide enrichment of the element $l$ at the evolutionary time $t$. Any of these chemical elements must obey a conservation law of this kind:

$\frac{\partial \rho^{l}}{\partial t}+\nabla \cdot\left(\rho^{l} \boldsymbol{v}\right)=\dot{\rho}^{l}$,

where $\rho^{l}$ represents the mass density of the $l$-th element, $v$ the fluid velocity and $\dot{\rho}^{l}$ is the mass return rate due to the contribution of SNeII, SNeIa and winds from IMS.

For massive and intermediate-mass stars we adopt the yields of Meynet \& Maeder (2002) (hereafter MM02) since they seem to better reproduce the chemical composition of IZw18 (Recchi et al. 2004). It is however worth noticing that the MM02 calculations do not take into consideration later phases of the stellar evolution (in particular the third dredgeup and the hot-bottom burning phase), and may underestimate the production of primary elements, in particular nitrogen. Although the 1-D simulations of Herwig (2004) predict 
a very efficient third dredge-up, Marigo (2003) showed that, assuming variable molecular opacities, the efficiency of the hot-bottom burning can significantly decrease, in particular for the more massive asymptotic giant branch stars. The yields of MM02, at variance with previous models, are obtained from self-consistent complete simulations of the stellar evolution and most of the primary nitrogen production stems from the physical effect of rotation. The importance of these new implications on the chemical evolution of galaxies has not yet been fully tested. Moreover, MM02 models are the ones which better reproduce the $\log (\mathrm{N} / \mathrm{O})$ and $\log (\mathrm{N} / \mathrm{Fe})$ ratios in the solar neighbourhood, as well as the solar abundance of helium (Chiappini et al. 2003b) and they also help to explain the relatively high $\log (\mathrm{N} / \mathrm{O})$ and $\log (\mathrm{N} / \mathrm{Fe})$ recently found in very metal-poor stars (Chiappini et al. 2005). A discussion on the effect of a different choice of stellar yields can be found in Sect. 4.1 .

We adopt the MM02 model with $Z=0.004$ and $v_{\text {ini }}=$ $300 \mathrm{~km} \mathrm{~s}^{-1}$. This metallicity is the one which better reproduces the CMD of NGC 1569 in the work of A05. For consistency reasons we adopt it as the metallicity of the stellar population. A05 used the Padova tracks (Fagotto et al. 1994) which are, in the low metallicity range, only calculated for $Z=0.004$ and $Z=0.0004$. Therefore A05 can just rule out a metallicity as low as $Z=0.0004$. We cannot therefore exclude a metallicity slightly lower than $Z=0.004$, in particular for old stellar populations. Therefore the nitrogen and carbon production from old stars should be considered as an upper limit. This compensates, at least qualitatively, the fact that, since MM02 do not follow the whole evolution of the star, the final nitrogen production might be larger than tabulated from these authors. The IMF slope of NGC 1569 is well constrained by the mass/luminosity ratio and is close to the Salpeter slope (Sternberg 1998). We therefore adopt a single-slope Salpeter IMF between 0.1 and $60 M_{\odot}$.

We assume, consistently with Recchi et al. (2004) a low thermalization efficiency for SNeII and a much larger value for SNeIa. This assumption is justified by the fact that stars are always born in molecular clouds, irrespective of the SF history of the galaxy. The first exploding SNe (the SNeII) have to get rid of cold and dense material before contributing to the total ISM energy budget, therefore they can loose a significant fraction of the mechanical energy produced by the explosion, at variance with what happens for SNeIa. In our simulations the SF is supposed to take place in an area of $200 \times 200 \mathrm{pc}^{2}$ (the field investigated by A05), therefore both energy sources (SNeII, SNeIa and winds from IMS) and mass return are concentrated inside this region. Greggio et al. (1998) performed observations with the WFPC2 onboard HST (where each WF covers a region of $80^{\prime \prime} \times 80^{\prime \prime}$ and the PC covers an area of $35^{\prime \prime} \times 35^{\prime \prime}$ ) and found that, outside the planetary camera, there is a limited amount of stars. In fact, the density of the detected stars in the PC is about 30 times higher than in each WF. Therefore, most of the SF in NGC 1569 has occurred in the central region and assuming an area of $200 \times 200 \mathrm{pc}^{2}$ for the input of energy and mass is a rather safe assumption, although A05 cannot exclude weak episodes of SF outside the investigated area.
The model contains also a metallicity-dependent cooling function (according to the tabulated values of Böhringer \& Hensler 1989) and the thermal conduction. The central resolution of the grid is $5 \mathrm{pc}$; the grid size expands logarithmically with a size ratio between adjacent zones of $\sim 1.03$.

\subsection{Star formation and gas mass}

As described in Sect. 2, the stellar population in NGC 1569 is dominated by stars younger than a few tens Myrs (Anders et al. 2004), although the presence of older stars has been inferred (Vallenari \& Bomans 1996; Greggio et al. 1998).

We therefore adopt two possible SF histories for NGC 1569. The first is a single burst of star formation, lasting for 25 Myr. The SF rate calculated by Greggio et al. (1998) for a Salpeter IMF is $0.5 M_{\odot} \mathrm{yr}^{-1}$. A weaker SF rate for the present burst $\left(0.13 M_{\odot} \mathrm{yr}^{-1}\right)$ has been found in A05. In this set of models, given the uncertainties of this value, we keep the SF rate as a free parameter. The duration of this SF episode, according to Anders et al. (2004) has been assumed to be 25 Myr. We have assumed three possible SF rates for these models: one with $0.1 M_{\odot} \mathrm{yr}^{-1}$ (model NGC - 1), one with $0.3 M_{\odot} \mathrm{yr}^{-1}$ (model $\mathrm{NGC}-2$ ) and one with $0.5 M_{\odot} \mathrm{yr}^{-1}$ (model NGC -3 ). In all models, the initial gas content of the galaxy is $10^{8} M_{\odot}$. For the model NGC -1 , each SP has therefore a mass of $10^{4} M_{\odot}$. Also for this relatively small SP, it is reasonable to assume a Salpeter IMF between 0.1 and $60 M_{\odot}$ and neglect statistical fluctuations. If we adopt the formulation of Weidner \& Kroupa (2004) and a theoretical upper stellar mass of $150 M_{\odot}$, the derived upper stellar mass cut-off for a star cluster of $10^{4} M_{\odot}$ is $\sim 100 M_{\odot}$ (see Weidner \& Kroupa 2004, their Fig. 5).

In the second set of models, we adopt the prescriptions of A05 described in Sect. 2, namely 3 episodes of SF. The first happened between 600 and $300 \mathrm{Myr}$ ago at a rate of $0.05 M_{\odot} \mathrm{yr}^{-1}$. This episode is followed by a period of inactivity of $150 \mathrm{Myr}$ and then by a second episode lasting $110 \mathrm{Myr}$ at a SF rate of $0.04 M_{\odot} \mathrm{yr}^{-1}$. After a short quiescent period (3 Myr) the last episode of SF started. The onset of this episode is therefore $37 \mathrm{Myr}$ ago, lasting until $13 \mathrm{Myr}$ ago (24 Myr of duration in total, consistent with the estimates of Anders et al. 2004) at a rate of $0.13 M_{\odot} \mathrm{yr}^{-1}$. Since in this case the SF rate is no longer a free parameter, we wish to explore the effect of a different initial ISM distribution. Since there are still uncertainties about the total gas mass of NGC 1569 (see e.g. Stil \& Israel 2002; Mühle et al. 2003) one has the freedom to test different values of the initial mass and to see which one is more appropriate to reproduce the characteristics of NGC 1569. In particular, we consider a "light" model (model NGC -4 ), in which the total galactic $\mathrm{H}$ I mass at the beginning of the simulation is $\sim 10^{8} M_{\odot}$ and a model with a factor of $\sim 2$ more gas initially present inside the galaxy (model NGC - 5). Finally, we consider a model in which a longer first episode of SF is considered. This episode lasts $1.7 \mathrm{Gyr}$, at a rate of $0.01 M_{\odot} \mathrm{yr}^{-1}$. The intermediate and the young episodes are the same as in model NGC -4 . This SF history still reproduces the CMD of NGC 1569 in a satisfactory way (see A05 and Sect. 2). Table 1 summarizes the parameters adopted to model NGC 1569. 
Table 1. Parameters for the NGC 1569 models.

\begin{tabular}{ccccc}
\hline \hline Model & SF episodes & SF rate $\left(M_{\odot} \mathrm{yr}^{-1}\right)$ & SF duration $(\mathrm{Myr})$ & $M_{\text {gas }}\left(M_{\odot}\right)$ \\
\hline NGC -1 & 1 & 0.1 & 25 & $10^{8}$ \\
NGC -2 & 1 & 0.3 & 25 & $10^{8}$ \\
NGC -3 & 1 & 0.5 & 25 & $10^{8}$ \\
NGC -4 & 3 & $0.05 ; 0.04 ; 0.13$ & $300 ; 110 ; 24$ & $10^{8}$ \\
NGC -5 & 3 & $0.05 ; 0.04 ; 0.13$ & $300 ; 110 ; 24$ & $1.8 \times 10^{8}$ \\
NGC -6 & 3 & $0.01 ; 0.04 ; 0.13$ & $1700 ; 110 ; 24$ & $10^{8}$ \\
\hline
\end{tabular}

\section{Chemical and dynamical evolution of NGC 1569}

\subsection{Single episode of star formation}

In this section we describe the results of the models in which a single burst of star formation has been considered, namely NGC -1 , NGC -2 and NGC -3 (see Table 1).

From the dynamical point of view, the model NGC -3 injects a large amount of energy into the ISM of the galaxy. This energy suffices to unbind a too large fraction of the gas in a short timescale so that at the end of the simulation more than $70 \%$ of the gas is lost by galactic winds. On the other hand, the model NGC - 1 with a much milder SF retains almost all the gas, showing no evidences for galactic winds. This disagrees with observations, since NGC 1569 shows evidences of the presence of outflowing gas (see Introduction). For the model NGC -2 , a galactic wind of moderate intensity starts at $t \sim 45$ Myr.

In Fig. 1 we plot the evolution of oxygen (upper panel) and $\mathrm{N} / \mathrm{O}$ ratio (lower panel) for these three models in comparison with the observations of Kobulnicky \& Skillman (1997). For the model NGC -1 , in spite of the fact that no oxygen is lost through galactic winds, the final oxygen content, due to the low number of SNeII, is by far not enough to justify the observed $12+\log (\mathrm{O} / \mathrm{H})$. On the other hand, model NGC -3 produces a final oxygen abundance closer (but still $\sim 0.2$ below) to the observed value. At the end of the starbursting phase, the oxygen abundance is larger than the observations, but at an age of $\sim 35$ Myr a metal-enriched galactic wind ensures a significant reduction of the $\mathrm{O}$ content of the galaxy. In the first few tens of Myrs, the nitrogen produced by intermediate-mass stars is not able to match the N/O ratio observed in NGC 1569. This ratio, however, increases with time due to the delayed production of nitrogen, reaching values close to the observations $\sim 80 \mathrm{Myr}$ after the onset of the burst (see Fig. 1, lower panel). At that time oxygen has already dropped below the observed value. Model NGC - 2 shows an intermediate behaviour. At an age of 35-40 Myr it reproduces the oxygen content of NGC 1569, but at this phase of the evolution, IMS have not significantly contributed to the global chemical enrichment of the galaxy, therefore the $\mathrm{N} / \mathrm{O}$ ratio is severely underestimated. After the onset of the galactic wind ( $t \sim 45 \mathrm{Myr}$ ), the oxygen content begins to decrease.

The N/O ratio of model NGC -2 and NGC -3 after the onset of the galactic wind becomes larger than the value predicted for model NGC - 1 (unaffected by selective loss of metals). This implies that, at the beginning, the outflowing gas is enriched in elements produced by SNeII (in particular

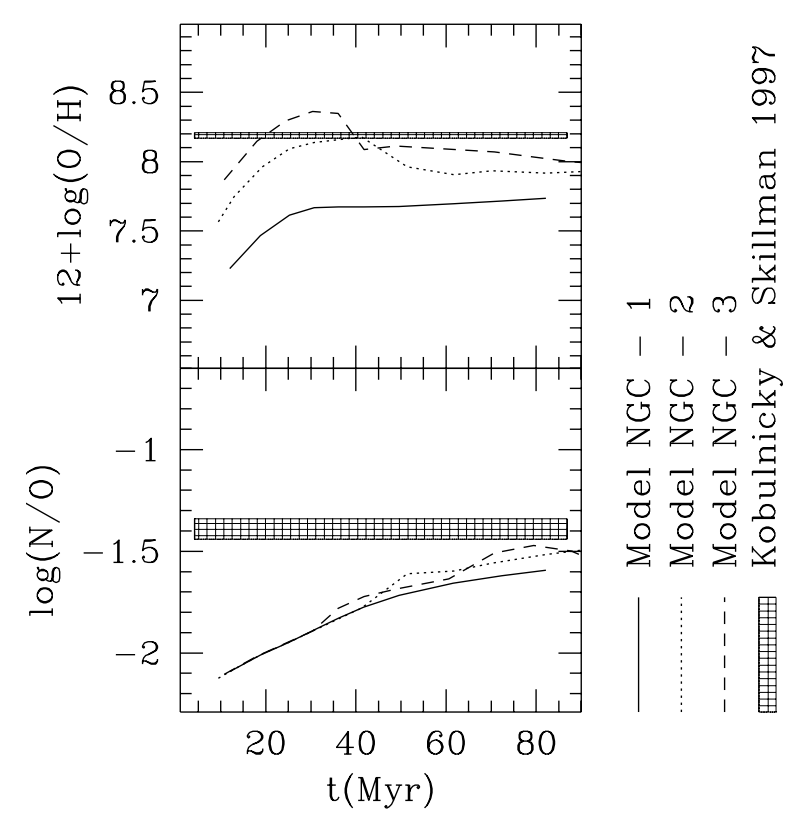

Fig. 1. Evolution of $12+\log (\mathrm{O} / \mathrm{H})$ (upper panel) and $\log (\mathrm{N} / \mathrm{O})$ (lower panel) for a NGC 1569 model with a single episode of SF at a rate of $0.1 M_{\odot} \mathrm{yr}^{-1}$ (model NGC - 1; solid lines), $0.3 M_{\odot} \mathrm{yr}^{-1}$ (model NGC - 2; dotted lines) and $0.5 M_{\odot} \mathrm{yr}^{-1}$ (model NGC -3 ; dashed lines). The superimposed shaded areas indicate the observed values found by Kobulnicky \& Skillman (1997), with error-bars.

$\alpha$-elements), in agreement with the observations of Martin et al. (2002; see also below in this section).

In summary, fine-tuning the SF rate in order to fit at the same time the oxygen abundance and the gas content of NGC 1569 is not easy, since a low SF rate can be insufficient to match the observed $\mathrm{O} / \mathrm{H}$ and a higher $\mathrm{SF}$ rate can drive a too powerful galactic wind, able to unbind a too large fraction of gas initially present inside the galaxy.

It is worth reminding that the yields of MM02 might underestimate the primary nitrogen production from IMS. Although, as pointed out in Sect. 3.2, this effect might be compensated by the fact that the adopted metallicity of the stellar populations $(Z=0.004)$ should be considered as an upper limit, it is worth briefly discussing the effect of a different choice of yields, in particular from IMS. Although the set-up is different, our simulations of the evolution of IZw18 (Recchi et al. 2004) give us a hint about the differences in N/O produced by different sets of IMS yields. In particular comparing Figs. 7 and 10 of Recchi et al. (2004), we obtain that the calculations of van den Hoek \& Groenewegen (1997) (together with the Woosley \& Weaver (1995) calculations for massive stars) would produce a final 
$\mathrm{N} / \mathrm{O} \sim 0.2-0.3$ dex larger than the one predicted by using the MM02 models. A similar result has been obtained by Chiappini et al. (2003b) by simulating the chemical evolution of the Milky Way and of the BCD galaxies. Looking at Fig. 1 we notice that a different choice of IMS yields would therefore produce, at the end of the simulation ( $\sim 80$ Myr after the onset of the starburst), a N/O consistent with the observed values of Kobulnicky \& Skillman (1997). However, at this age, the oxygen content of the galaxy would be underestimated for any of the considered models. Since the yields of oxygen are more reliable than those of nitrogen, from the upper panel of Fig. 1 one should argue that the only acceptable age of the burst is $35-40 \mathrm{Myr}$, provided that the SF rate is larger than $0.3 M_{\odot} \mathrm{yr}^{-1}$. At this age, the predicted N/O is $\sim 0.4-0.5$ dex below the observed values. Therefore, also the yields of van den Hoek \& Groenewegen would not match the observations. It is thus absolutely reasonable to implement the MM02 yields.

\subsection{The $X$-ray emitting gas}

As anticipated in the introduction, Martin et al. (2002) were able to estimate the metallicity of the hot X-ray emitting gas in the galactic wind. This information completes the puzzle of understanding the metal enrichment. Even if the single-burst models are not able to account for the chemical and morphological properties of NGC 1569, it is nonetheless interesting to calculate the metallicity of the hot gas (i.e. of the gas with temperatures larger than $0.3 \mathrm{keV}$ ) and to compare it with the derived values of Martin et al. (2002). This comparison is shown in Fig. 2 for the NGC - 3 model. At the moment of the onset of a galactic wind, the oxygen abundance of the hot phase is already solar. The arrows drawn in the plot represent the estimated oxygen content of the galactic wind of NGC 1569 (best fit; upper and lower limits). The oxygen abundance of the hot gas increases continuously in this phase, since after $50 \mathrm{Myr}$ massive stars are still exploding and releasing oxygen into the interstellar medium. At later times, however, the oxygen composition begins to decrease due to the larger fraction of pristine gas ablated from the supershell and entrained in the galactic wind and due to the lower amount of gas heated up. The $[\mathrm{O} / \mathrm{Fe}]$ ratio is initially larger than solar due to the fact that the break-out occurs when SNeIa are not yet releasing their energy and metals into the ISM. At later times, since the SNeIa expel their products from the galaxy very easily (Recchi et al. $2001)$, the $[\mathrm{O} / \mathrm{Fe}]$ ratio decreases. The observations of Martin et al. (2002) however point towards a galactic wind dominated by $\alpha$-elements, therefore an outflow probably is still triggered by SNeII.

\subsection{Three episodes of star formation}

As shown in the previous section, single SF bursts of short duration are not able to account for the global properties of NGC 1569. According to observationally derived SF we therefore describe the evolution of models with the SF as a gasping process, occurring in 3 different episodes as explained in Sect. 3.2.

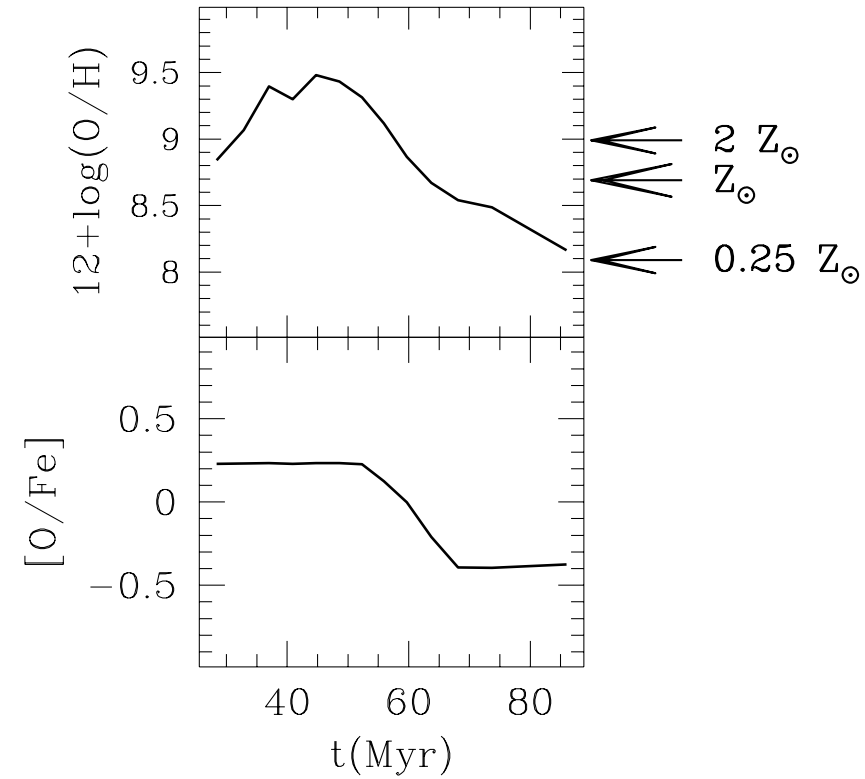

Fig. 2. Evolution of $12+\log (\mathrm{O} / \mathrm{H})$ (upper panel) and $[\mathrm{O} / \mathrm{Fe}]$ abundance ratio (lower panel) for the hot gas for the model NGC -3 . The arrows indicate the oxygen abundance of the galactic wind inferred by Martin et al. (2002).

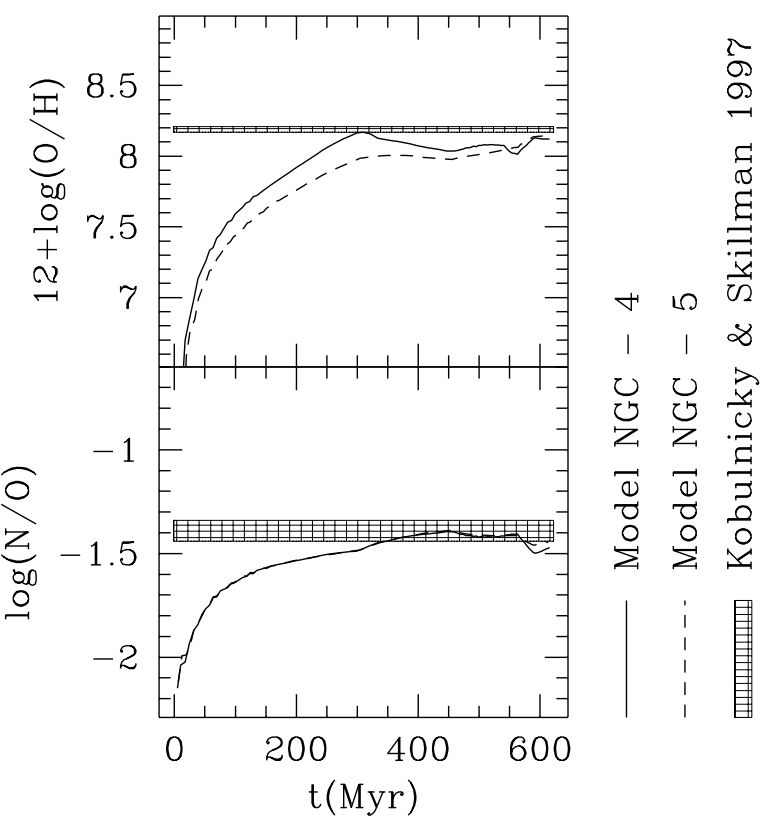

Fig. 3. Evolution of $12+\log (\mathrm{O} / \mathrm{H})$ (upper panel) and $\log (\mathrm{N} / \mathrm{O})$ (lower panel) for two NGC 1569 models in which the A05 SF history is implemented: model NGC -4 (solid line) and NGC - 5 (dashed line). The superimposed dashed areas represent the observed values found for NGC 1569 (Kobulnicky \& Skillman 1997).

In Fig. 3 we show the evolution of oxygen (upper panel) and $\log (\mathrm{N} / \mathrm{O})$ (lower panel) for the models NGC -4 (solid lines) and NGC - 5 (dashed lines) and we compare them with the abundances derived by Kobulnicky \& Skillman (1997) (dashed areas). At the end of the simulations (after $\sim 600 \mathrm{Myr}$ ), the oxygen is reproduced nicely by the model NGC -5 and also model NGC -4 is close to the observed value. It is worth noticing that the outflow created by the pressurized gas is very 


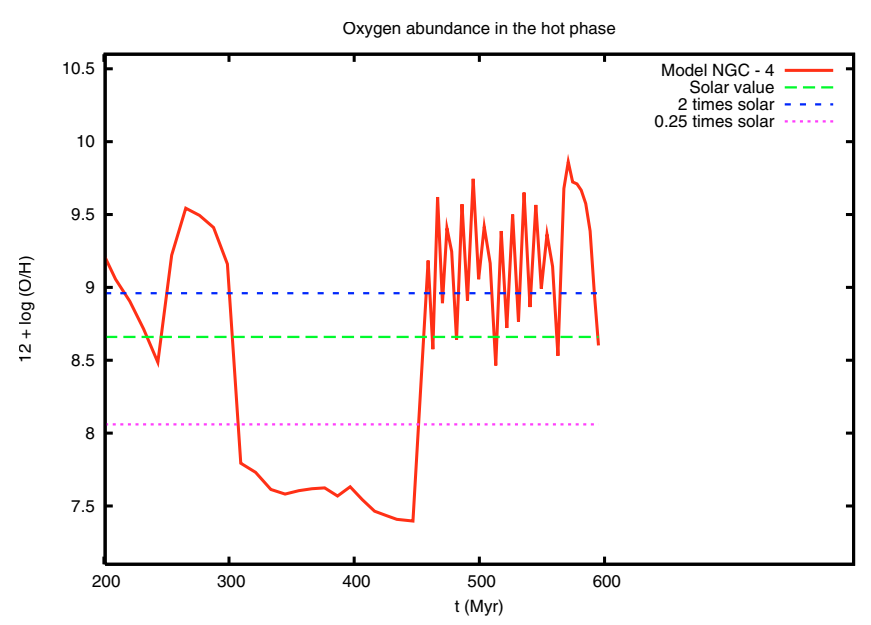

Fig. 4. Evolution of $12+\log (\mathrm{O} / \mathrm{H})$ for the hot gas for the model NGC - 4 (see Table 1). The oxygen abundance of the hot phase inferred by Martin et al. (2002) is indicated by 3 horizontal lines: dotted line $\left(Z=0.25 Z_{\odot}\right)$, long-dashed line $\left(Z=1 Z_{\odot}\right)$ and short-dashed line $\left(Z=2 Z_{\odot}\right)$.

weak in the NGC - 5 model and of moderate intensity for the model NGC -4 . The fraction of oxygen lost through the galactic wind is larger in the light model (the one with lower total mass). In the first hundreds of Myr the oxygen abundance predicted by the model NGC - 4 is larger, since it is diluted by a smaller amount of hydrogen. At later times, however, the $\mathrm{O}$ abundance predicted by this model slightly decreases with time, since some oxygen is expelled from the galaxy.

The final $\log (\mathrm{N} / \mathrm{O})$ predicted by these models are -1.47 (model NGC - 4) and - 1.44 (model NGC - 5). These values slightly underestimate the observations of Kobulnicky \& Skillman (1997), but are still reasonably close to it, considering the observational errors $(0.05 \mathrm{dex})$.

For model NGC - 4 (the light model), we also have plotted the oxygen content of the hot phase and we have compared it with the values inferred by Martin et al. (2002). This comparison is shown in Fig. 4. As one can see, the power generated by the bursts of SF is able to heat a significant amount of oxygen, whereas, during the quiescent periods, the fraction of hot gas drops considerably. Due to the poor spatial resolution of our simulation at large radii, the oxygen abundance of the hot phase shows large oscillations. The average value of $\mathrm{O} / \mathrm{H}$ during the last $150 \mathrm{Myr}$ is slightly larger than $2 Z_{\odot}$, whereas, at the end of the simulation, this value drops to $\sim Z_{\odot}$ which is the best fit of Martin et al. (2002). However, due to the strong variation of $\mathrm{O} / \mathrm{H}$ with time, we cannot consider it as a firm prediction of our model.

We describe now the evolution of the model NGC -6 , namely a model in which the first episode of SF lasts $1.7 \mathrm{Gyr}$, at a rate of $0.01 M_{\odot} \mathrm{yr}^{-1}$ and the other two episodes are identical to the ones adopted in models NGC -4 and NGC -5 . The total energy released in this episode of SF is slightly larger than in the previous cases. Moreover, the continuous input of energy affects the gas distribution in front of the bubble along the $z$ direction and, although the energy input rate is pretty mild, at $\sim 600 \mathrm{Myr}$ a galactic wind arises and is sustained until the end of the simulation. This also produces a significant metal

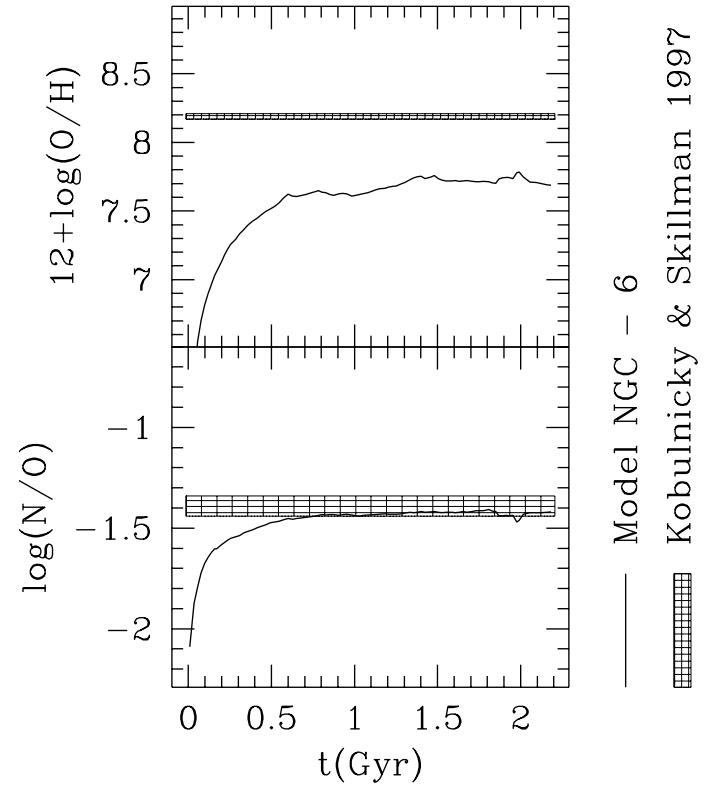

Fig. 5. Evolution of $12+\log (\mathrm{O} / \mathrm{H})$ and $\log (\mathrm{N} / \mathrm{O})$ for the model NGC -6 (see Table 1$)$.

loss and, at the end of the simulation, the oxygen content of the galaxy is $\sim 0.4-0.5$ dex below the observations (see Fig. 5). This model is therefore less suited than models NGC -4 and NGC 5 to explain the observed chemical composition of NGC 1569.

Finally, we briefly mention the effect of a different IMF choice. We ran a model in which the IMF slope is flatter than Salpeter $(x=0.85$ instead of $x=1.35)$. As described in A05, a flatter IMF is still marginally consistent with the observed CMD diagram of NGC 1569. Although the Salpeter IMF is able to nicely reproduce the mass/luminosity ratio in NGC 1569 (Sternberg 1998) it is worth analyzing the results of a different IMF slope. As expected, a model with a flatter IMF produces a very large fraction of massive stars. The overproduction of oxygen is partially compensated by the fact that, due to the larger energy input from SNeII, the galactic wind is more intense and therefore the fraction of ejected $\mathrm{O}$ is larger. At the end of the simulation the oxygen is overestimated by $\sim 0.2$ dex compared to observations. The nitrogen is underproduced (due to the minor fraction of IMS) and the final $\log (\mathrm{N} / \mathrm{O})$ is $0.4-0.5 \mathrm{dex}$ below the observations. As shown in Recchi et al. (2004), a steeper IMF slope would underproduce oxygen and overestimate $\log (\mathrm{N} / \mathrm{O})$. Therefore, the choice of a Salpeter slope seems to be the safest one also from a chemodynamical point of view.

\subsection{The cooling of freshly produced metals}

As one can see in Fig. 3. the effect of the last, more intense burst of SF (occurring at an evolutionary time of $~ 560 \mathrm{Myr}$ ) is almost unrecognizable in the NGC -5 model and also in the NGC -4 model a very mild increase in oxygen by $\sim 0.1$ dex (and a relative drop in N/O) is visible. This is due to the fact that the hot cavity carved by the previous episodes of SF is very large and a significant fraction of the freshly produced gas is already carried outside the galaxy. Therefore, the freshly produced metals are released in a hot and rarefied environment 


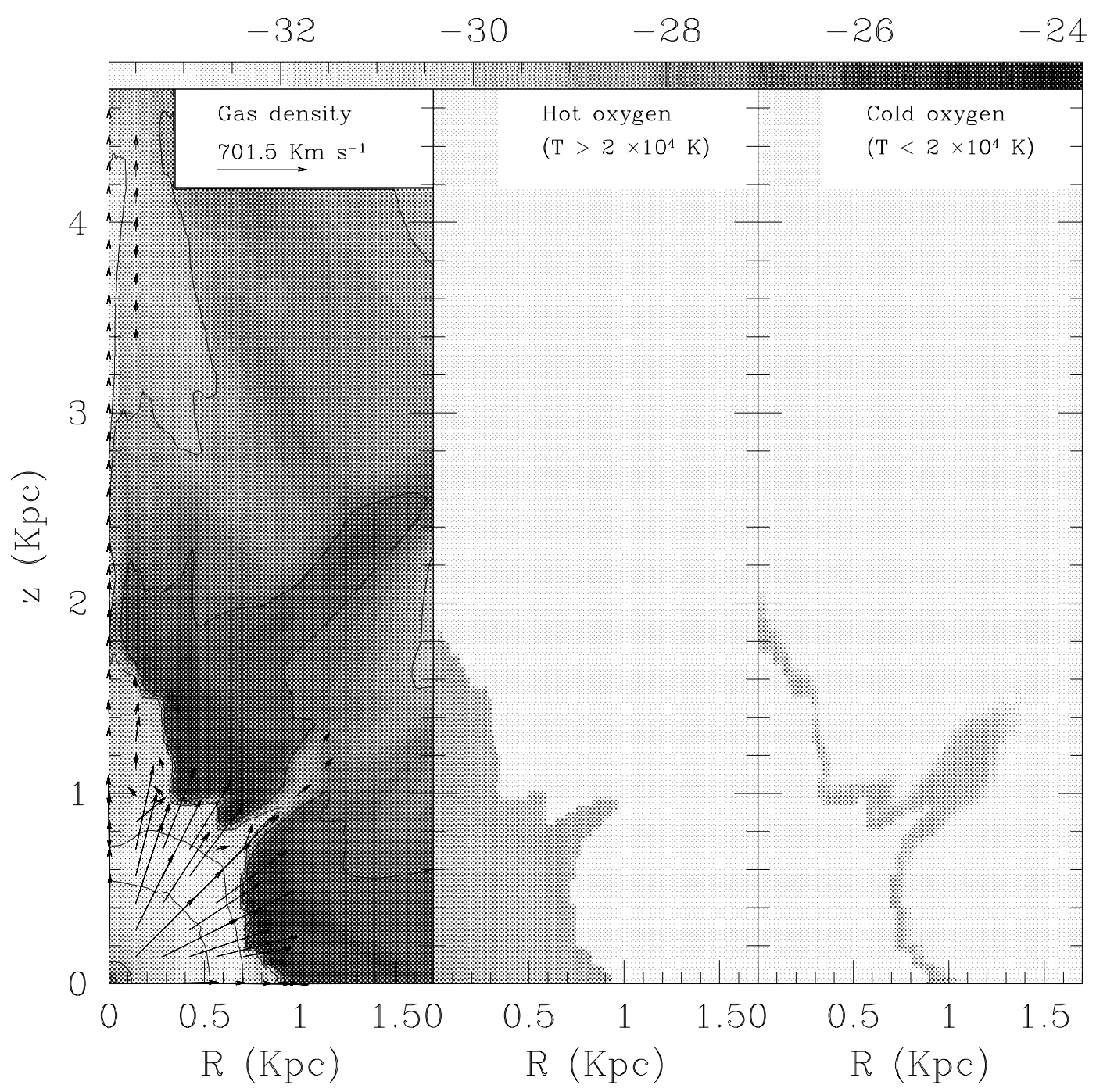

Fig. 6. Density contours and velocity fields for model NGC $-4 \sim 15 \mathrm{Myr}$ after the onset of the last episode of SF (left panel). Also plot are the distribution of cold $\left(T<2 \times 10^{4} \mathrm{~K}\right)$ and hot $\left(T \geq 2 \times 10^{4} \mathrm{~K}\right)$ oxygen at the same evolutionary time (left and central panel, respectively).

and their cooling time-scales are very long. To visualize this effect, we consider the evolution of model NGC $-4 \sim 15 \mathrm{Myr}$ after the onset of the last burst of SF. In Fig. 6 we plot the density contour and velocity field (left panel) of this model, together with the distribution of cold $\left(T<2 \times 10^{4} \mathrm{~K}\right)$ and hot ( $T \geq 2 \times 10^{4} \mathrm{~K}$ ) oxygen (left and central panel, respectively). It is evident that only a negligible fraction of freshly produced oxygen has cooled down. Most of the cold oxygen is located in a tongue-like structure at $R \sim 1 \mathrm{kpc}, z \sim 1 \mathrm{kpc}$ that has been produced by Kelvin-Helmholtz instabilities resulting from the shear of the hot gas when flowing through the funnel visible at $R \sim 0.7 \mathrm{kpc}, z \sim 0.8 \mathrm{kpc}$ in the density distribution plot (left panel). The bulk of the freshly produced oxygen is in a hot phase (central panel) and will stay hot until the end of the simulation.

The fate of the oxygen produced in the previous episodes of SF is significantly different. To demonstrate that, in Fig. 7 the same plot (density distribution, distribution of cold and hot oxygen) is shown, but for the oxygen produced by the first episode of SF by the model NGC - 4 after an evolutionary time of $\sim 80 \mathrm{Myr}$. As one can see, in this case the evolution of the superbubble is still relatively slow (due to the low SF rate) and a significant fraction of the freshly produced oxygen can be pushed close to the cavity walls, where eddies (particularly evident is an eddy at $R \sim 0.1 \mathrm{kpc}, z \sim 0.35 \mathrm{kpc}$ ), thermal instabilities and thermal conduction are able to broaden the interface between the hot cavity and the cold shell, resulting in a relatively fast cooling of a significant amount of freshly produced metals. The fact that the chemical elements produced by the last episode of SF do not cool down (and therefore do not contribute to the chemical composition of the $\mathrm{H}$ II regions) helps to explain why most of the H II regions in NGC 1569 show similar chemical compositions (Kobulnicky \& Skillman 1997). This composition is in fact mostly due to the metal production of the older episodes of SF, which have had enough time to cool down and to disperse throughout the galaxy.

\subsection{The intergalactic medium enrichment}

It is now well established that the iron content in the Intracluster Medium (ICM) is pretty large, ranging from $\sim 1 / 5$ to about half solar, with a mean value around $1 / 3$. The $[\alpha / \mathrm{Fe}]$ 


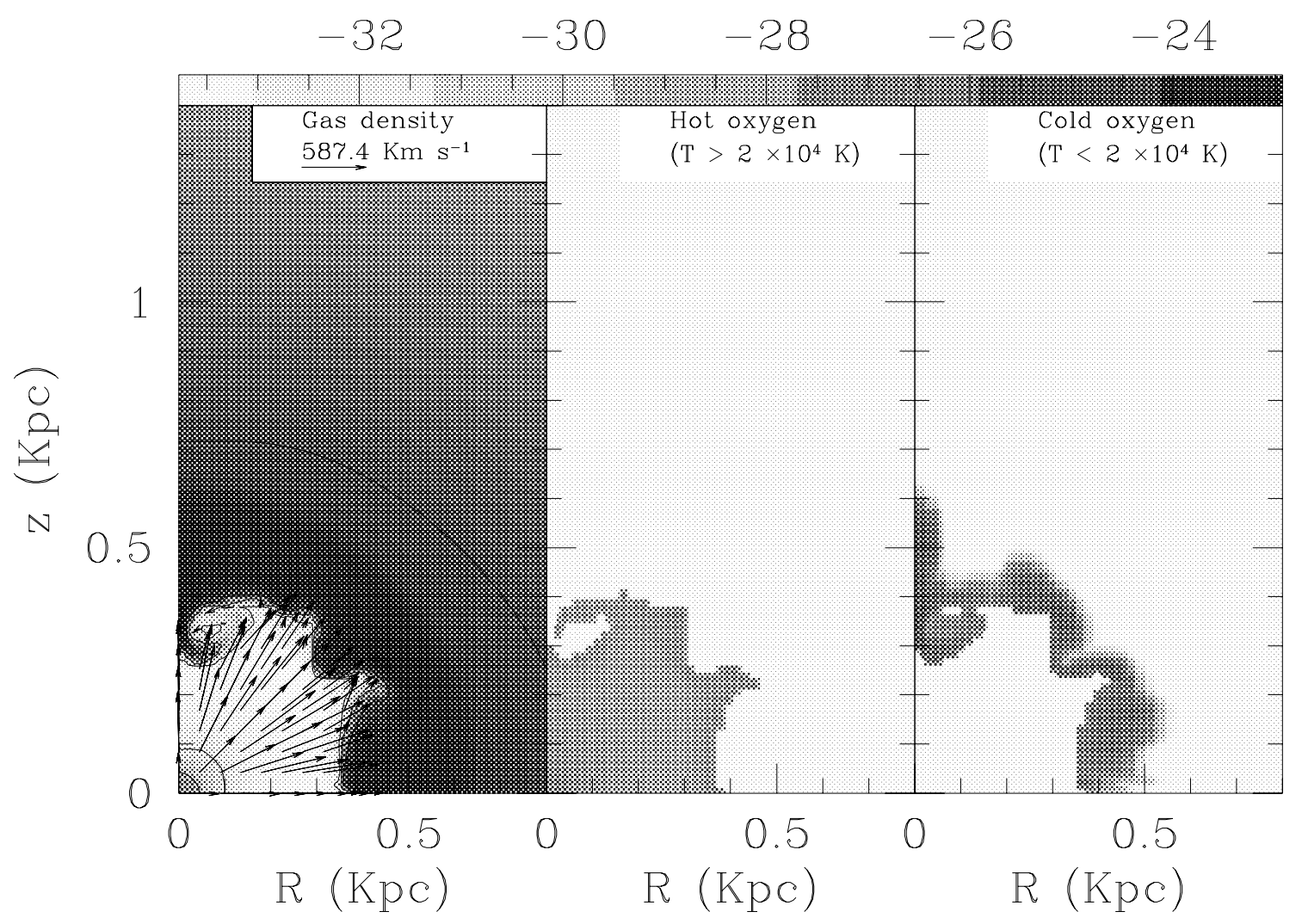

Fig. 7. As Fig. 6 but $\sim 80 \mathrm{Myr}$ after the onset of the first episode of SF.

ratios are close to solar (Renzini 1997), or even undersolar ([O/Fe] -0.3) for M 87 (Gastaldello \& Molendi 2002; Matsushita et al. 2003). Some dependence on the cluster metallicity or temperature may also be present (Finoguenov et al. 2000; Loewenstein 2004). We thus need to invoke a mechanism able to enrich iron in the ICM to a level at least comparable to the enrichment of $\alpha$-elements.

A first assumption is that Pop. III stars (Ostriker \& Gnedin 1996) or proto-galaxies at very high $z$ (Mori et al. 2002) can increase the metallicity of the ICM to the levels observed nowadays. However, observations have established that the enrichment of the ICM at $z \sim 3$ is very low, about two orders of magnitude lower than the metal content reached at $z \sim 0$ (see Aguirre et al. 2001 and references therein). Most of the ICM enrichment should have occurred between $z \sim 2$ and $z \sim 1$, since there is no significant evolution of the Fe metallicity out to $z \sim 1$ (Tozzi et al. 2003). Thus, these pre-galactic object can enrich the ICM but only to a very low level so that most of the ICM enrichment has to come from galaxies.

We can only imagine two processes able to enrich the ICM: ram pressure stripping and galactic winds. Ram pressure stripping may be very effective in removing the gas in dwarf galaxies (Mori \& Burkert 2000; Marcolini et al. 2003), and its effect can be significant also in larger disk galaxies (Roediger \& Hensler 2005). However, cosmological simulations by Aguirre et al. (2001) show that ram pressure stripping can only explain $\sim 1 / 12$ of the metals observed in the ICM. Moreover, ram pressure stripping affects mostly the external part of the discs (Roediger \& Hensler 2005), thus involving regions in which the iron enrichment might be negligible. Galactic winds instead are able to reproduce both the metallicity and the thermal history of the ICM (Pipino et al. 2002).

In the literature it is often claimed that the SNII products (thus in particular $\alpha$-elements) should dominate the gas ejected through galactic winds (Bradamante et al. 1998; D'Ercole \& Brighenti 1999, among others). The observation of the outflow metallicity in NGC 1569 seems to confirm this conclusion. The same happens also in our models, since, as we have seen in Figs. 2 and 4, during and immediately after the bursts of SF a very large amount of oxygen is transported out of the galaxy through the galactic wind. As time goes on, in the models in which a galactic wind is formed, the wind is progressively enriched in iron-peak elements, due to the minor work required to escape the galactic potential well once a funnel has been carved (see also Recchi et al. 2001, 2004). This can produce a significant release of iron from dwarf galaxies to the ICM, which stops only when the energy provided by SNeIa is no longer able to sustain the galactic wind and the center of the galaxy is refilled with cold gas. This happens a few hundreds Myrs after the end of the SF activity (Recchi \& Hensler, in preparation).

It is however worth pointing out that, although the number of dwarf galaxies in clusters is very large, the cluster luminosity (and mass) is dominated by giant elliptical galaxies. Gibson \& Matteucci (1997) demonstrated that, under conservative hypotheses, dwarf galaxies can provide at most $\sim 15 \%$ of the ICM gas through galactic winds, whereas the giant ellipticals are responsible for $\sim 20 \%$ and the remaining $\sim 65 \%$ being of 
primordial origin. Indeed also giant elliptical galaxies can provide a significant iron ICM enrichment. This depends on the interplay between the energy input from SNeIa and the mass restored to the ISM from dying stars. If the secular type Ia SN rate decreases faster than the rate of mass return from IMS, the energy of SNeIa can sustain a supersonic galactic wind (expelling a significant fraction of iron) for several Gyr. This wind will later on turn to a subsonic wind and eventually an inflow (Ciotti et al. 1991; Renzini et al. 1993; Pipino et al. 2002, 2005). The reverse sequence applies in case of rates of SNeIa milder than the rates of mass return from dying stars. In this case early inflows are eventually followed by galactic winds (Loewenstein \& Mathews 1987).

\subsection{The evolution of $\log (\mathrm{N} / \mathrm{O})$ and the "plateau" problem}

Nitrogen determinations are available for a bulk of DIrrs and BCD galaxies (see e.g. Pagel et al. 1992; Masegosa et al. 1994; Kobulnicky \& Skillman 1996; van Zee 1997; Izotov \& Thuan 1999, Vílchez \& Iglesias-Páramo 2003; Skillman et al. 2003, among others).

Nitrogen results from $\mathrm{CNO}$ processing of $\mathrm{O}$ and $\mathrm{C}$ during hydrogen burning. This can happen either from $\mathrm{O}$ and $\mathrm{C}$ originally present in the star (secondary $\mathrm{N}$ production), or from $\mathrm{O}$ and $\mathrm{C}$ produced in situ and dredged up to the $\mathrm{H}$-burning layer.

The behaviour of the N/O ratio as a function of $\mathrm{O} / \mathrm{H}$ has provided the main observational constraint to the processes for $\mathrm{N}$ formation (secondary and/or primary). Assuming instantaneous recycling (i.e. assuming that all the stars with masses larger than $1 M_{\odot}$ die instantaneously), if $\mathrm{N}$ is produced in a secondary way, it increases proportionally to the metal content of the galaxy and the track in the $\mathrm{N} / \mathrm{O}$ vs. $\mathrm{O} / \mathrm{H}$ diagram is a straight line at a $45^{\circ}$ slope. If instead the nitrogen is produced in a primary way, it evolves independently of the $\mathrm{O}$ content of the galaxy (Tinsley 1980; Matteucci 1996). However, the effect of the delayed production of $\mathrm{N}$, due to its progenitors, must also be taken into account. Observations of metal-poor $(\log (\mathrm{O} / \mathrm{H}) \lesssim-4) \mathrm{H}$ II regions in dwarf galaxies (van Zee et al. 1997; Izotov \& Thuan 1999) have revealed that N/O is almost independent of $\mathrm{O} / \mathrm{H}$ and this is what is expected if $\mathrm{N}$ is produced in a primary way. On the other hand, for high metallicity $\mathrm{H}$ II regions, N/O increases almost linearly with $\mathrm{O}$, indicating that most of $\mathrm{N}$ is produced in a secondary way. A large scatter $( \pm 0.3 \mathrm{dex})$ in the $\mathrm{N} / \mathrm{O}$ vs. $\mathrm{O} / \mathrm{H}$ diagram is present at large metallicities, and this has been interpreted as the consequence of the delayed production of $\mathrm{N}$, resulting in a "saw-tooth" evolution in this diagram (Pilyugin 1993; Marconi et al. 1994; Olofsson 1995). At lower O the scatter identified by Izotov \& Thuan (1999) is very small. The conclusion of these authors is that these metal-poor galaxies are undergoing their first burst of SF and the observed $\mathrm{N}$ comes only from primary production in massive stars. Observations of DLAs (see e.g. Cénturion et al. 2003, and references therein) show a significant population of objects with N/O lying at $0.7-0.8$ dex below the plateau indicated by Izotov \& Thuan (1999). If $\mathrm{N}$ is produced in a primary way in massive stars, as invoked for metal-poor BCDs, it would be hard to explain this population of objects with very low $\mathrm{N}$ content (but see also Izotov et al. 2001). Moreover, none of the nucleosynthetic prescriptions available in literature are able to reproduce the observed N/O ratio by means of massive stars alone (Recchi et al. 2002).

We have collected the most recent data available in literature about $\mathrm{O}$ and $\mathrm{N}$ abundances in very metal-poor galaxies (with $12+\log (\mathrm{O} / \mathrm{H}) \leq 8.2)$. In Fig. 8 we plot these data, together with the evolution in the $\mathrm{N} / \mathrm{O}$ vs. $\mathrm{O} / \mathrm{H}$ plane of the bursting NGC -3 (solid line) and the gasping NGC - 4 (longdashed line) models. For comparison, we also plot two models describing the evolution of IZw18, following two instantaneous bursts of SF (dotted line) and a gasping SF history, with a weak episode of SF lasting $270 \mathrm{Myr}$, a quiescent period of $10 \mathrm{Myr}$ and a more intense burst lasting $5 \mathrm{Myr}$ (short-dashed line). These models have been comprehensively described in Recchi et al. (2002) and Recchi et al. (2004), respectively. In the bursting model (dotted line), only the evolution after the onset of the second burst is plotted. In this sample of data, we can notice the presence of a considerable spread, at variance with the results of Izotov \& Thuan (1999). Moreover, Kennicutt \& Skillman (2001) have pointed out that the observed narrow dispersion in the Izotov \& Thuan (1999) observations itself is difficult to understand given the associated uncertainties in reddening corrections, the ionization correction factor, and the estimated temperature in the $\mathrm{O}^{+}$zone. The "plateau" at log (N/O) $\simeq-1.6$ needs therefore more statistics in order to be firmly established, also considering that, in order to have a homogeneous sample of objects, in this figure we have not plotted the N/O of DLA systems, whose inclusion would arise in a considerable increase of the scatter. It is however worth pointing out that Henry et al. (2000) performed simulations in which they were able to fit the trend of N/O for BCDs with a very low $\mathrm{SF}$ rate, whereas a higher $\mathrm{SF}$ rate were invoked to explain the DLAs with low oxygen and low N/O.

The comparison between the observed data and the evolutionary paths allows us to understand how the $\mathrm{N} / \mathrm{O}$ ratio changes in the first phases of the evolution of the galaxy. However, we have to bear in mind that, as shown by Chiappini et al. (2003a), the observed data in the N/O vs. O/H diagram do not give us any information about time evolution. They are simply the present-day status of each single galaxy. As we have seen in the previous sections, model NGC -3 (single-burst model) is unable to account for the $\mathrm{O} / \mathrm{H}$ and $\mathrm{N} / \mathrm{O}$ ratio at the same time, whereas model NGC - 4 gets very close to the observations of Kobulnicky \& Skillman (1997). A vertical line indicates also the moment of the onset of the last episode of SF. This happens when the $12+\log (\mathrm{O} / \mathrm{H})$ has already reached 8.13 and little or no variations of the chemical composition of the galaxy happen afterwards. The last burst of SF is therefore unable to modify the chemical composition of the galaxy observed in the H II region, due to the fact that the metals produced by the last SF episode are injected in a too hot phase and cannot contribute to the total chemical budget of the galaxy (see Sect. 4.4). Models describing bursting and gasping SF, for both IZw18 and NGC 1569, show similar patterns: a bursting model produces huge variations of the chemical composition of the galaxy in short time-scales, whereas the gasping model 


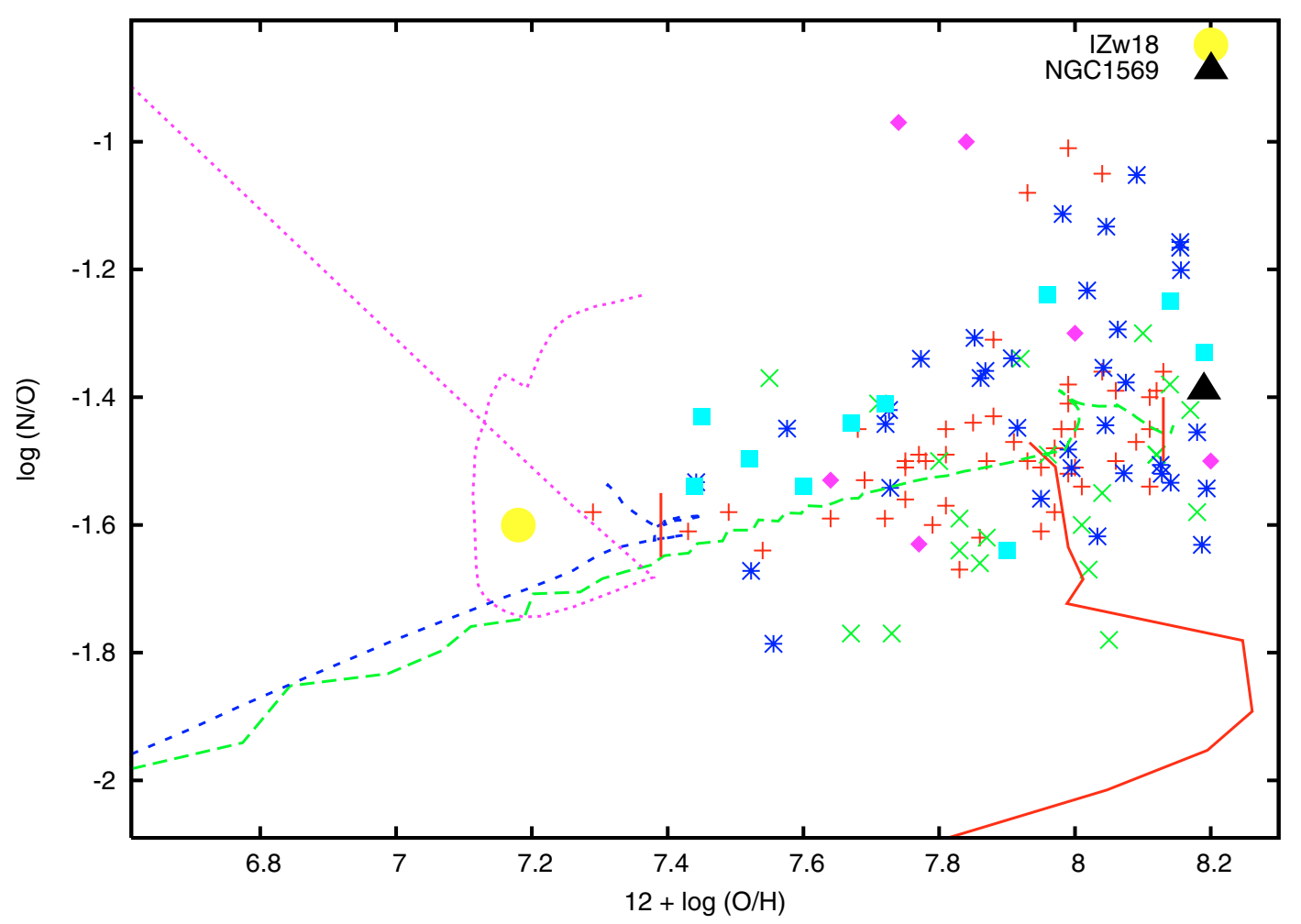

Fig. 8. $\log (\mathrm{N} / \mathrm{O})$ vs. $12+\log (\mathrm{O} / \mathrm{H})$ in metal-poor BCD and DIrr galaxies. Filled circle is the IZw18 value calculated by Izotov \& Thuan (1999), whereas the filled triangle is the NGC 1569 value from Kobulnicky \& Skillman (1997). Pluses are other galaxies detected by Izotov \& Thuan (1999). The collection of data by van Zee et al. (1997) is shown by crosses. The measurements of BCD galaxies by Kobulnicky \& Skillman (1996) are indicated by asterisks. Filled rhombs represent the values tabulated by Vílchez \& Iglesias-Páramo (2003). The other data points (filled squares) are taken from different sources. Also shown is the evolution in the N/O vs. O/H plane of two NGC 1569 models: model NGC -3 (solid line) and NGC - 4 (long-dashed line). For comparison, we also plot two model reproducing the evolution of IZw18 after 2 instantaneous bursts (dotted line) and after a gasping star formation derived by Aloisi et al. (1999) (short-dashed line). These last two models are taken from Recchi et al. (2002) and Recchi et al. (2004), respectively. A vertical line indicates, for model NGC - 4 and for the gasping IZw18 model the onset of the last burst of SF.

predicts a mild increase of oxygen and of N/O and a stabilization of the chemical composition at later times (also for the gasping IZw18 model we have plotted a vertical line at the onset of the last burst of SF). It is also visually clear from this plot that gasping models are much more adequate to explain the similar $\mathrm{N} / \mathrm{O}$ ratios in metal-poor galaxies. If the plateau suggested by Izotov \& Thuan (1999) is real, we thus have to invoke the scenario of weak and continuous episodes of SF in order to explain the observations. Episodes of SF older (and weaker) than the ones considered in our simulations are not ruled out by A05. When considering episodes of SF older than $\sim 2 \mathrm{Gyr}$, their nature (bursting or gasping) become less relevant, since the abundance ratios are dominated by the SF occurring in the last $\sim 2 \mathrm{Gyr}$ (Romano et al. 2005b). Finally, in spite of a difference in the oxygen abundance of $\sim 1$ dex between IZw18 and NGC 1569, the difference in (N/O) is $\sim 0.2$, which might again indicate a mild nitrogen production in some long-lasting episode of star formation, as it happens in the model NGC - 4 (long-dashed line in Fig. 8).

\subsection{Models with a big infalling cloud}

As described in the introduction, extended H I clouds surround NGC 1569 (Stil \& Israel 1998). In particular, a series of gas clumps in the southern part of the galaxy, probably connected with the H I arm, led Mühle et al. (2005) to the hypothesis that these clumps can be attributed to the debris of a tidally disrupted big cloud infalling towards NGC 1569. The mass of this complex is difficult to assess. The lower limit given by Mühle et al. (2005) is $1.2 \times 10^{7} M_{\odot}$ (the sum of the mass of all the detected groups of clouds), but some H I could have been already accreted. If this complex is similar to the HVCs in the Local Group, one has to expect masses larger than a few $10^{7} M_{\odot}$ (Blitz et al. 1999; de Heij et al. 2002). From a chemical point of view, the expected effect of an infalling cloud is to dilute the gas in the galaxy, allowing for a reduction of the metallicity without altering the abundance ratios (Köppen \& Hensler 2005). In reality, the coexistence of hot and cold gas leads to interfaces due to heat conduction where from analytical estimates under "normal" conditions cold gas should suffer evaporation. From a dynamical point of view, this leads to massloaded flows (Dyson \& Hartquist 1987) in which the specific energy is reduced so that an outflow can be hampered. More realistic hydrodynamical simulations of self-gravitating interstellar clouds could, however, show that their disruption is prevented (Vieser \& Hensler 2005). Moreover, an infalling cloud produces ram pressure which acts like a "cap" for the expansion of the superbubble. 


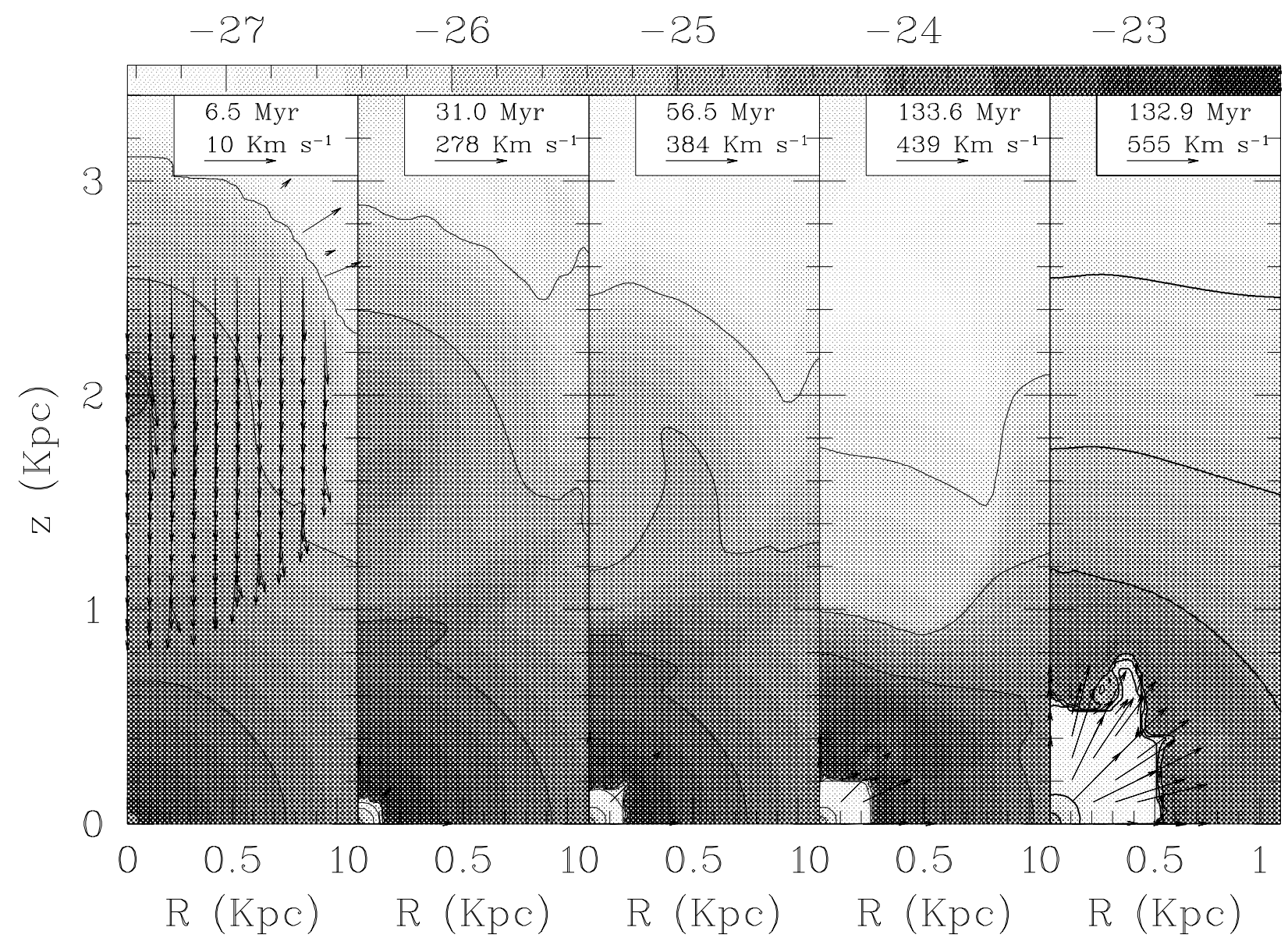

Fig. 9. Density contours and velocity fields for model NGC $-4+B C$ (heavy cloud) at four different epochs (evolutionary times are labelled in the box on top of each panel). In the last panel the density contours and velocity fields for the reference model NGC -4 at a similar evolutionary time as the last plot of model NGC $-4+\mathrm{BC}$ are shown. The logarithmic density scale is given in the strip on top of the figure. In order to avoid confusion, velocities with values lower than $1 / 10$ of the maximum value (indicated for each panel in the upper right box) are not drawn.

In order to study the effect of a big cloud infalling towards the galaxy, we assume a mass of $2 \times 10^{7} M_{\odot}$. The initial position of the cloud is $2 \mathrm{kpc}$ away from the center of the galaxy along the polar axis (due to the assumed axial symmetry of the system, this is the only reasonable initial configuration). The chosen density profile of the cloud is a power law, with a slope of -1.7 (de Heij et al. 2002). The infalling velocity of this cloud is $10 \mathrm{~km} \mathrm{~s}^{-1}$, similar to the local sound speed, and its radius is $1 \mathrm{kpc}$. The metallicity of the cloud is set to 0 . Two possible SF scenarios have been analyzed: a single burst of SF, as in model NGC -3 , therefore with a SF rate of $0.5 M_{\odot} \mathrm{yr}^{-1}$ and a model, analogous to model NGC - 4 in which the implemented $\mathrm{SF}$ history is the one inferred by A05. These models are named $\mathrm{NGC}-3+\mathrm{BC}$ and $\mathrm{NGC}-4+\mathrm{BC}$, respectively. For the model NGC $-4+B C$ we also consider the possibility of a lighter infalling cloud, namely a cloud with a total mass of $10^{7} M_{\odot}$ (half of the standard value).

In Fig. 9 snapshots of the evolution of the model NGC$4+\mathrm{BC}$ (with the standard $2 \times 10^{7} M_{\odot}$ cloud) during the first $\sim 130$ Myr are shown. In the left panel, the big cloud and its infalling motion is clearly visible. It is also worth noticing that we do not put the cloud in pressure equilibrium with the surrounding ISM, therefore the cloud tends to expand towards the regions of minor pressure. The pressure of the infalling cloud limits the expansion of the superbubble and shrinks it, mostly in the polar direction. In the second-last plot shown in Fig. 9 the cavity has an extension of $\sim 250 \times 200 \mathrm{pc}^{2}$. For comparison, we also plot in Fig. 9 (right panel) the density contours and velocity fields for the reference model NGC -4 at an evolutionary time similar to the last one considered for the model NGC $-4+\mathrm{BC}$. As one can see, the cavity carved by the ongoing episode of SF is much larger in this case $\left(\sim 500 \times 600 \mathrm{pc}^{2}\right)$ and the density in front of the superbubble is also much smaller, since in the model NGC $-4+\mathrm{BC}$ the big cloud has compressed the gas in front of the superbubble along the $z$ direction up to densities of the order of $\sim 10^{-23} \mathrm{~g} \mathrm{~cm}^{-3}$. The effect of the infall is therefore to hamper the development of a galactic wind and to confine the superbubble well inside the galactic region. Only in the model NGC $-4+B C$ with light cloud a moderate galactic wind is still present and some gas (and metals) is lost. Moreover, the cloud on its path towards the galaxy sweeps up and drags some halo gas present in the outer regions. The final gas mass inside the galaxy is therefore larger than the initial one.

Also the model NGC $-3+\mathrm{BC}$, in spite of the large SF rate, does not experience large outflows and the mass of the H I gas increases with time. It is worth noticing that, as we have pointed out in the previous sections, NGC 1569 does show 
Model NGC-3+BC Model NGC-4+BC

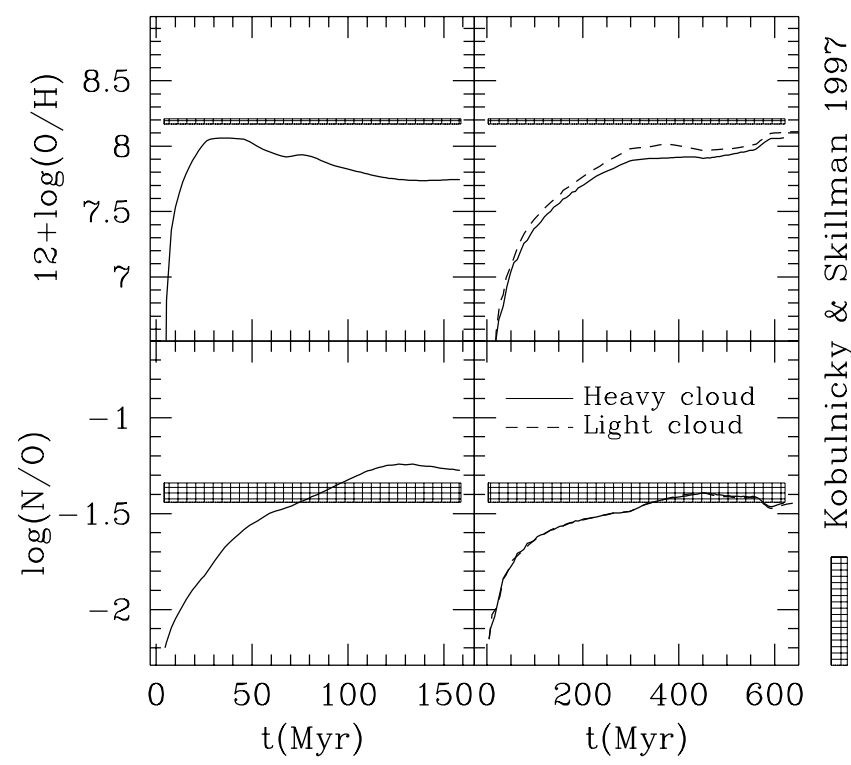

Fig. 10. Evolution of $12+\log (\mathrm{O} / \mathrm{H})$ (upper panels) and $\log (\mathrm{N} / \mathrm{O})$ (lower panels) for two NGC 1569 models in which the infall of a big cloud towards the center of the galaxy is taken into consideration. Left panels show a model in which a single burst of SF, lasting $25 \mathrm{Myr}$, is considered (model NGC $-3+\mathrm{BC}$ ), whereas in the right panels the evolution of a model implementing the SF rate inferred by A05 is shown (model NGC $-4+\mathrm{BC}$ ). In this case we consider two possible masses of the big cloud: $10^{7} M_{\odot}$ (light cloud; dashed line) and $2 \times$ $10^{7} M_{\odot}$ (heavy cloud; solid line).

a prominent outflow (Martin et al. 2002), at variance with the results of these simulations. Therefore, we either have to consider a larger input of energy into the system, or have to consider a different infall direction of the cloud. Indeed, observations show that this H I complex seems to wrap around the disk of NGC 1569 and to approach the galaxy from the western side (Mühle et al. 2005). Such an infall geometry is however impossible to reproduce with a 2-D simulation in cylindrical coordinates. Although the 2-D symmetry can only account for a vertical infall along the $z$-axis, one can already learn from this kind of simulations to what extent the pressure of the cloud hampers the expansion of the superbubble. Moreover, since there are no extensive calculations available in the literature about chemodynamical evolution of galaxies with infall, it is extremely interesting to study what changes does this infall produce on the global chemical budget of the galaxy (see e.g. Köppen \& Hensler 2005).

The evolution of oxygen and $\log (\mathrm{N} / \mathrm{O})$ for these models is shown in Fig. 10. Since almost no metals are lost through the galactic wind, due to the hampering effect of the infalling cloud, the oxygen produced by the various episodes of SF is mostly retained by the galaxy and mixes with the surrounding ISM. Only for the model NGC $-4+B C$ with light cloud (dashed lines in the right plots) a weak galactic wind produces a drop in the oxygen content at $\sim 450 \mathrm{Myr}$. In the model NGC $-3+\mathrm{BC}$, in which the oxygen is produced only in the first $\sim 50 \mathrm{Myr}$, the dilution effect due to the infalling gas is more evident. Part of the unprocessed gas dragged by the cloud mixes with the metals released by the burst of $\mathrm{SF}$, reducing the global metallicity of the galaxy. The final $\log (\mathrm{N} / \mathrm{O})$ of model $\mathrm{NGC}-4+\mathrm{BC}$ is consistent with the observations, whereas this model slightly underestimates the final oxygen content of the galaxy (by $\sim 0.1-0.15$ dex depending on the mass of the big cloud). The oxygen abundance in the model NGC $-3+\mathrm{BC}$ always remains more than 0.2 dex below the observations.

\section{Conclusions}

By means of a 2-D hydrodynamical code, we have studied the chemical and dynamical evolution of model galaxies resembling NGC 1569, a gas-rich dwarf galaxy in the aftermath of an intense burst of SF. We have considered either one episode of SF of short duration (bursting SF), or more complex behaviours, in which the galaxy has experienced long-lasting episodes of SF in the past, separated from the last, more intense burst, by short periods of inactivity (gasping SF).

Models with a bursting SF are generally unable to account for the chemical and morphological properties of this object, since they either severely underproduce $\mathrm{O}$, or inject too much energy into the system, enough to unbind a too large fraction of the gas initially present in the galaxy.

The best way to reproduce the chemical composition of NGC 1569 is therefore assuming long-lasting, continuous episodes of SF of some hundreds Myrs of age and a recent and more intense short burst. Adopting the SF prescriptions derived from the comparison of the color-magnitude diagrams with synthetic ones (A05) we produce results in good agreement with the observations, if the yields of MM02 are implemented. A simulation in which a big cloud is falling towards the center of the galaxy along the polar axis produces also results in good agreement with the observed chemical composition of NGC 1569, but the cloud inhibits almost completely the formation of a galactic wind, in contradiction with observations.

In most models with gasping SF, the final chemical composition of the galaxy reflects mostly the chemical enrichment from old stellar populations. In fact, if the first episodes of SF are powerful enough to create a galactic wind or to heat up a large fraction of the gas surrounding the star forming region, the metals produced by the last burst of SF are released in a too hot medium to be observed or are directly ejected from the galaxy through the wind. They do not have the chance to pollute the surrounding medium and contribute to the chemical enrichment of the galaxy. This results confirms the finding of Martin et al. (2002) that most of the oxygen produced in the last episode of SF is in the hot, X-ray emitting phase.

Acknowledgements. S.R. acknowledges generous financial support from the Alexander von Humboldt Foundation and Deutsche Forschungsgemeinschaft (DFG) under grant HE 1487/28-1. S.R. would also like to thank Stefanie Mühle and Donatella Romano for their suggestions and fruitful discussions, Monica Tosi for providing a new CMD of IZw18 in advance of publication and Stefan Hirche for careful reading the first version of the manuscript. We finally thank the anonymous referee for thoughtful comments and suggestions, which have greatly improved the paper. 


\section{References}

Aguirre, A., Hernquist, L., Schaye, J., et al. 2001, ApJ, 561, 521 Aloisi, A., Tosi, M., \& Greggio, L. 1999, AJ, 118, 302

Aloisi, A., Clampin, M., Diolaiti, E., et al. 2001, AJ, 121, 1425

Anders, E., \& Grevesse, N. 1989, Geochim. Cosmochim. Acta, 53, 197

Anders, P., de Grijs, R., Fritze-v. Alvensleben, U., \& Bissantz, N. 2004, MNRAS, 347, 17

Angeretti, L., Tosi, M., Greggio, L., et al. 2005, AJ, 129, 2203 (A05)

Aparicio, A., \& Gallart, C. 1995, AJ, 110, 2105

Arnone, E., Ryan, S. G., Argast, D., Norris, J. E., \& Beers, T. C. 2005, A\&A, 430, 507

Arp, H., \& Sandage, A. 1985, AJ, 90, 1163

Asplund, M., Grevesse, N., \& Sauval, A. J. 2005, in Cosmic Abundances as Records of Stellar Evolution and Nucleosynthesis, ed. F. N. Bash, \& T. G. Barnes (San Francisco: ASP), ASP Conf. Ser., 336, 25

Babul, A., \& Rees, M. J. 1992, MNRAS, 255, 346

Blitz, L., Spergel, D. N., Teuben, P. J., Hartmann, D., \& Burton, W. B. 1999, ApJ, 514, 818

Böhringer, H., \& Hensler, G. 1989, A\&A, 215, 147

Bradamante, F., Matteucci, F., \& D'Ercole, A. 1998, A\&A, 337, 338

Centurión, M., Molaro, P., Vladilo, G., et al. 2003, A\&A, 403, 55

Chiappini, C., Romano, D., \& Matteucci, F. 2003a, MNRAS, 339, 63

Chiappini, C., Matteucci, F., \& Meynet, G. 2003b, A\&A, 410, 257

Chiappini, C., Matteucci, F., \& Ballero, S. K. 2005, A\&A, 437, 429

Ciotti, L., D’Ercole, A., Pellegrini, S., \& Renzini, A. 1991, ApJ, 376, 380

de Heij, V., Braun, R., \& Burton, W. B. 2002, A\&A, 391, 67

de Marchi, G., Clampin, M., Greggio, L., et al. 1997, ApJ, 479, L27

D'Ercole, A., \& Brighenti, F. 1999, MNRAS, 309, 941

Dyson, J. E., \& Hartquist, T. W. 1987, MNRAS, 228, 453

Ellis, R. 1997, ARA\&A, 35, 389

Fagotto, F., Bressan, A., Bertelli, G., \& Chiosi, C. 1994, A\&A, 105, 29

Finoguenov, A., David, L. P., \& Ponman, T. J. 2000, ApJ, 544, 188

Gallagher, J. S., Mould, J. R., de Feijter, E., et al. 1996, ApJ, 466, 732

Gastaldello, F., \& Molendi, S. 2002, ApJ, 572, 160

Gibson, B. K., \& Matteucci, F. 1997, ApJ, 475, 47

Greggio, L., Tosi, M., Clampin, M., et al. 1998, ApJ, 504, 725

Heckman, T. M., Dahlem, M., Lehnert, M. D., et al. 1995, ApJ, 448, 98

Henry, R. B. C., Edmunds, M. G., \& Köppen, J. 2000, ApJ, 541, 660

Herwig, F. 2004, ApJ, 605, 425

Israel, F. P. 1988, A\&A, 194, 24

Izotov, Y. I., Schaerer, D., \& Charbonnel, C. 2001, ApJ, 549, 878

Izotov, Y. I., \& Thuan, T. X. 1999, ApJ, 511, 639

Izotov, Y. I., \& Thuan, T. X. 2004, ApJ, 616, 768

Kennicutt, R. C., Jr., \& Skillman, E. D. 2001, AJ, 121, 1461

Kobulnicky, H. A., \& Skillman, E. D. 1996, ApJ, 471, 211

Kobulnicky, H. A., \& Skillman, E. D. 1997, ApJ, 489, 636

Köppen, J., \& Hensler, G. 2005, A\&A, 434, 531

Kunth, D., \& Östlin, G. 2000, A\&ARv, 10, 1

Lilly, S. J., Tresse, L., Hammer, F., Crampton, D., \& Le Fevre, O. 1995, ApJ, 455, 108

Loewenstein, M., 2004, in Origin and Evolution of the Elements, ed. A. McWilliam, \& M. Rauch (Cambridge University Press), Carnegie Observatories Astrophys. Ser., 4425

Loewenstein, M., \& Mathews, W. G. 1987, ApJ, 373, 445

Marcolini, A., Brighenti, F., \& D'Ercole, A. 2003, MNRAS, 345, 1329

Marconi, G., Matteucci, F., \& Tosi, M. 1994, MNRAS, 270, 35

Marconi, G., Tosi, M., Greggio, L., \& Focardi, P. 1995, AJ, 109, 173
Marigo, P. 2003, in CNO in the Universe, ed. C. Charbonnel, D. Schaerer, \& G. Meynet, ASP Conf. Ser., 304, 312

Martin, C. L. 1998, ApJ, 506, 222

Martin, C. L., Kobulnicky, H. A., \& Heckman, T. M. 2002, ApJ, 574, 663

Masegosa, J., Moles, M., \& Campos-Aguliar, A. 1994, ApJ, 420, 576

Matsushita, K., Finoguenov, A., \& Böhringer, H. 2003, A\&A, 202, 21

Matteucci, F. 1996, Fund. Cosm. Phys., 17, 283

Matteucci, F., \& Recchi, S. 2001, ApJ, 558, 351

Meynet, G., \& Maeder, A. 2002, A\&A, 390, 561 (MM02)

Momany, Y., Held, E. V., Saviane, I., et al. 2005, A\&A, 439, 111

Mori, M., \& Burkert, A. 2000, ApJ, 538, 559

Mori, M., Ferrara, A., \& Madau, P. 2002, ApJ, 571, 40

Mühle, S., Klein, U., Wilcots, E. M., \& Hüttermeister, S. 2003, ANS, 324,40

Mühle, S., Klein, U., Wilcots, E. M., \& Hüttermeister, S. 2005, AJ, 130,524

Olofsson, K. 1995, A\&A, 293, 652

Origlia, L., Leitherer, C., Aloisi, A., Greggio, L., \& Tosi, M. 2001, AJ, 122,815

Östlin, G. 2000, ApJ, 535, L99

Ostriker, J. P., \& Gnedin, N. Y. 1996, ApJ, 472, L63

Ott, J., Walter, F., \& Brinks, E. 2005, MNRAS, 358, 1423

Pagel, B. E. J., Simonson, E. A., Terlevich, R. J., \& Edmunds, M. G. 1992, MNRAS, 255, 325

Pilyugin, L. S. 1993, A\&A, 277, 42

Pipino, A., Matteucci, F., Borgani, S., \& Biviano, A. 2002, NewA, 7, 227

Pipino, A., Kawata, D., Gibson, B. K., \& Matteucci, F. 2005, A\&A, 434, 553

Reakes, M. 1980, MNRAS, 192, 297

Recchi, S., Matteucci, F., \& D'Ercole, A. 2001, MNRAS, 322, 800

Recchi, S., Matteucci, F., D’Ercole, A., \& Tosi, M. 2002, A\&A, 384, 799

Recchi, S., Matteucci, F., D’Ercole, A., \& Tosi, M. 2004, A\&A, 426, 37

Renzini, A. 1997, ApJ, 488, 35

Renzini, A., Ciotti, L., D’Ercole, A., \& Pellegrini, S. 1993, ApJ, 419, 52

Roediger, E., \& Hensler, G. 2005, A\&A, 433, 875

Romano, D., Tosi, M., \& Matteucci, F. 2005a, in Starbursts - From 30 Doradus to Lyman break galaxies, ed. R. de Grijs and R.M. Gonzalez Delgado (Dordrecht: Springer), Ap\&SS Library, 329, P67

Romano, D., Tosi, M., \& Matteucci, F. 2005b, MNRAS, accepted [arXiv: astro-ph/0510113]

Ruiz-Lapuente, P., \& Canal, R. 1998, ApJ, 497, L57

Searle, L., Sargent, W. L. W., \& Bagnuolo, W. G. 1973, ApJ, 179, 427

Skillman, E. D., Côté, S., \& Miller, B. W. 2003, AJ, 125, 610

Spite, M., Cayrel, R., Plez, B., et al. 2005, A\&A, 430, 655

Sternberg, A. 1998, ApJ, 506, 721

Stil, J. M., \& Israel, F. P. 1998, A\&A, 337, 64

Stil, J. M., \& Israel, F. P. 2002, A\&A, 392, 473

Tinsley, B. M. 1980, Fund. Cosm. Phys., 5, 287

Tosi, M., Greggio, L., Marconi, G., \& Focardi, P. 1991, AJ, 102, 951

Tozzi, P., Rosati, P., Ettori, S., et al. 2003, ApJ, 593, 705

Vallenari, A., \& Bomans, D. J. 1996, A\&A, 313, 713

van den Hoek, L. B., \& Groenewegen, M. A. T. 1997, A\&AS, 123, 305

van Zee, L., Haynes, M. P., \& Salzer, J. J. 1997, AJ, 114, 2479

Vieser, W., \& Hensler, G. 2005, A\&A, submitted

Vílchez, J. M., \& Iglesias-Páramo, J. 2003, ApJS, 145, 225

Weidner, C., \& Kroupa, P. 2004, MNRAS, 348, 187

Woosley, S. E., \& Weaver, T. A. 1995, ApJS, 101, 181 